\title{
Editorial
}

\section{Health care in the era of innovations}

In 1995, nearly two decades ago, Harvard Business School Professor Clayton Christensen coined the phrase "disruptive innovation" to describe new technologies that transform industries by bringing simplicity and affordability to products and processes that are complicated and high cost. The word has been used since then rather loosely to describe stumbling of a previously developed technology by a newer one. However, "Disruption" describes a process whereby a smaller company with fewer resources is able to successfully challenge established incumbent technology because of its simplicity, affordability and acceptability by the people. In fact, it focus on improving on some existing technology so that it also targets the overlooked segments at an economical price. Gradually it is being adopted by a larger segment of people and led to disruption of existing technology.

Healthcare is now at cusp of drastic changes fuelled by demand and emerging technologies. Biomedical engineers are working hard to take their model work from bench to bedside but are facing challenges. Scientists are unprepared and only focus on technology without a consideration for personal touch or interaction. This may prove to be disastrous as the efforts which have gone in designing the technology will not be acceptable by people and profession generally. Another challenge is the implementation which should stand by the regulatory hurdles. Here comes the quality. The regulatory bodies wish to ensure that the new product is safe, better, durable and comforms to survival of an individual to life time. That means that rigorous clinical trials are needed which judges the robustness of new technology to surpass the existing method.

It has now been felt by physicians and biomedical scientists that in order for an innovation to be successful it should have capability to empower patients. There is a need for harnessing technology for an end in human experimentation. For example, instead of doing a trial on animals and humans, we can design billions of simulations using computing and bioinformatics devices to reach same conclusion which are more fool proof. Moreover we can make use of artificial intelligence in research and biodesign. 
Emergence of 3D printing technology has open a new gateway for faster technology development bypassing the time consuming mathematical calculations.

The drug development can have a handshake with genomics to enable personalized medicine individualised to the need of each person.

In this issue of Annals one can feel the impact of such innovations.

Dr Sanjeev Misra 